\title{
The influence of Gas Type on a Thermal Mass Flowmeter Calibration Results
}

\author{
Oras ABBAS ${ }^{1,{ }^{*}}$, Fabrice Mouchel ${ }^{1}$ \\ ${ }^{1}$ Gas and liquid flow laboratory of Cherbourg -Trescal, France
}

\begin{abstract}
The Thermal Mass Flowmeters (TMFMs) have been used for more than twenty years in the industry for various uses, including sweeping, gas injection, and welding. Their operational behaviors are still not well understood by operators. TMFMs, could be used with multiple process gases, and sometimes, highly toxic gases and they are generally calibrated with nitrogen or air. In the case of using a surrogate gas for calibration, a correction factor ( $k$-factor) is required to estimate the flow of the process gas. Most of the time, these correction factors are indicated in the manufacturer's instructions manual. In the field of metrology, kfactor plays a significant role in calibration results. Therefore, it is important to understand the influence of gas type used in calibration on the results if the process gas is employed or a surrogate gas with the recommended $\mathrm{k}$-factor. The aim of this paper therefore is to discuss and evaluate the impact of using manufacturers $\mathrm{k}$-factor in place of the actual process gas during calibration. The tests results presented within this article are considered concerning within the field of metrology.
\end{abstract}

\section{Introduction}

The metrology of gas flow relies often upon the Gas massflow meters. Thermal Mass Flow-Meters (TMFMs) are essentially used to monitor and control gas flow in many manufacturing processes such as semiconductor manufacturers. These meters sense the flow by measuring the thermal transfer between a heated tube wall and the gas stream.

TMFMs are often used with multiple gases or highly explosive and toxic gases. Due to this fact, it is common practice to calibrate the instrument with a gas, such as air or nitrogen, and employ correction factors to estimate the flow with respect to the process gases. These correction factors are instrument specific and may vary by as much as $10 \%$ between instruments of different designs, errors in the correction factors can introduce significant errors to measurements that rely upon them. The uncertainty given is around $\pm 1 \%$ of $\mathrm{k}$-factor. Unfortunately, these factors are still not well understood and raise a question of reliability in metrology.

Additionally, it has been assumed that the correction factors may be a function of flow and not constant at all [1]. Errors in the measured flow are also incurred when the temperature or pressure of the gas differs from their calibrated values. Manufacturers usually report an estimated uncertainty due to these effects, but the accuracy of these estimates is unknown.

In this article we investigate the performance of TMFMs for a variety of gases (air and argon) under different scenarios. The accuracy of the TMFMs manufacturers calibration with air or argon as well as the operating conditions such as temperature and pressure are presented. For this purpose, a metrological study has been carried out, on six different TMFM's manufacturers with a flow range varying between $0.2-100 \mathrm{l}(\mathrm{n}) / \mathrm{min}$ (n: normal conditions of temperature and pressure, 273.15 Kelvin and $101325 \mathrm{~Pa})$.

\section{Operating principles of TMFMs}

TMFMs offer a significant potential in flow rate metering. They are used for accurate measurements of fluid (gaseous) flows in many different application areas. Their main advantage is the direct mass flow measurement without the need for additional density corrections as with volumetric flow meters, differential pressure and turbine or ultrasonic flow meters [2].

Fundamentally, the operating concept of TMFMs is based on heating of the fluid passing through the flowmeter. Any fluid passing through a hotter container will absorb heat. The flowmeter consists of two temperature probes placed at the inlet and the outlet. The flowmeter will measure the thermal power needed to maintain a constant temperature differential between these two probes. The flow sensor measures the mass flowrate as a function of temperature difference. This can be expressed according to first law of thermodynamics (heat in $=$ heat out, for no losses) for which the following formulae is applicable[3]:

$$
q m=\frac{(P-L) * f}{C_{p} *(\Delta T)}
$$

Where : $\mathrm{q} m$ is mass flow rate $\left(\mathrm{kg} . \mathrm{s}^{-1}\right) ; C_{p}$ is the specific heat $\left(\mathrm{J} \cdot \mathrm{kg}^{-1} \cdot \mathrm{K}^{-1}\right) ; \Delta T$ is the temperature difference $(K) ; P$ is the input power $(W) ; L$ is the conduction loss $(W) ; f$ is the proportionality factor of the meter (dimensionless).

There are generally two principles of thermal flowmeters: on-line principle and bypass principle [4]. The flowmeters used in this article are bypass flowmeters. This principle is based on the relationship between the

* Corresponding author: oras.abbas@trescal.com 
output voltage of sensors and heat transfer rate produced by the sensor itself and the gas flow in the pipe (Fig.1). In fact, the output voltage is influenced by the gas composition through its thermo-physical properties such as thermal conductivity, diffusivity, density, specific heat and dynamic viscosity. Gas properties depend on the operating temperature, pressure and the chemical composition [5].

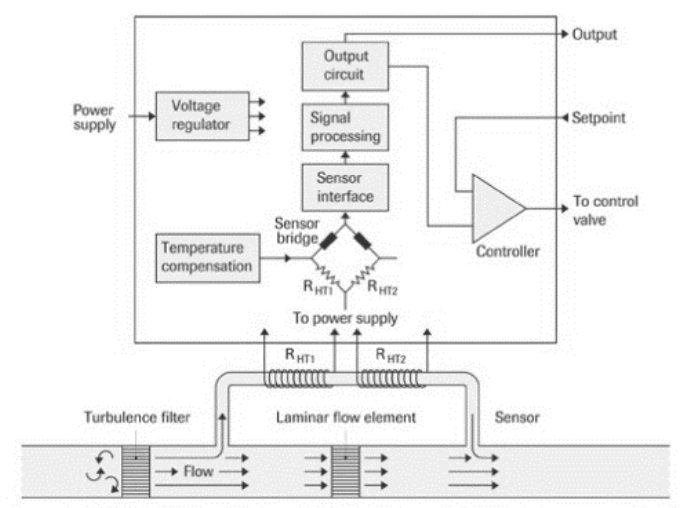

Fig. 1. Principle of Bypass Thermal Mass flowmeter (Bronkhost,2010)

Taking into consideration that the TMFMs are sometimes used with corrosive or hazardous gases, not all calibrations can be performed using the desired process gas. Therefore, it is essential to use a surrogate gas for calibration, i.e. air or nitrogen. In this case, it is necessary to know the k-factor value of the process gas. Each manufacturer of a TMFM's product is potentially able to supply a list of gas conversion factors or to refer to an appropriate database. The $\mathrm{k}$-factor for a mass flow is defined as[3]:

$$
k=\frac{C_{p, \text { ref }}}{C_{p, \text { Process }}}
$$

$k$ is the $k$-factor;

$C_{p, r e f}$ is the specific heat, at constant pressure, of the reference gas;

$C_{p, \text { Process }}$ is the specific heat, at constant pressure of the process gas;

\section{Material and methods}

\subsection{Calibration methodology}

The molbloc/molbox gas flow calibration systems are used for calibrating flow meters and controllers over a very broad spectrum of flow rates. The molbloc/molbox achieves high levels of stability and accuracy. This system has an operational range from less than $1 \mathrm{sccm}$ (standard cubic centimeter) to greater than $100 \mathrm{sl} / \mathrm{min}$ (s: standard conditions of temperature and pressure, 293.15 Kelvin and $101325 \mathrm{~Pa}$ ), with accuracy of $\pm 0.2 \%$ of reading in a variety of gases, and can be fully automated with COMPASS software. Molbloc is at the heart of the measurement system, it is mechanically stable with gravimetrically calibration. The molbloc/molbox flow measurement principle follows established laminar flow theory. The flow of a known gas in the laminar flow regime can be calculated from the flow path geometry and the gas pressure and temperature using Poiseuille law [6]:

$$
q m=\frac{\left(P_{1}-P_{2}\right) \cdot \rho_{(P, T)}}{\eta_{I P, T)}} * C_{G(R e)}
$$

$q m$ : mass flow $[\mathrm{kg} / \mathrm{s}]$

$P_{1}$ :upstream absolute pressure $[\mathrm{Pa}]$

$\mathrm{P}_{2}$ :downstream absolute pressure $[\mathrm{Pa}]$

$T$ :absolute temperature of gas $[\mathrm{k}]$

$\eta_{(P, T)}$ :dynamic gas viscosity under $P, T$ conditions [Pa $\left.\bullet \mathrm{s}\right]$ $C_{G(R e):}$ geometry of molbloc flow path, determined by gravimetric calibration $\left[\mathrm{m}^{3}\right]$

Re: Reynolds number

The mass flowrate is directly proportional to the pressure drop across the element. This allows molbloc/molbox to deliver flow readings in real time- as frequently as once per second with minimum uncertainty.

Cherbourg Gas Flow laboratory (France) has been Accredited since 2010 in accordance with ISO/IEC 17025. It is mainly equipped with four laminar flowmeters (Molbloc-L) and one sonic flowmeter (Molbloc-S) covering the flowing ranges $4.3 \mathrm{E}-7 \mathrm{~kg} . \mathrm{s}^{-1}$ (0.02 1/min) $-1.08 \mathrm{E}-2 \mathrm{~kg} . \mathrm{s}^{-1}(850 \mathrm{l} / \mathrm{min})$, utilising an uncertainty of 5,0E-3.Qm. Two different molbox are associated to the molblocs: molbox 1 and molbox RFM with manufacturer uncertainty $(0.2 \% ; 0.5 \%$ of reading $)$ respectively. These molboxes perform the data acquisition and processing functions necessary to determine the flow through a molbloc flow element that is connected to it.

The used calibration method is based on direct comparison between the Device Under Test (DUT) and one or more molbloc type flow meters in laminar or sonic regime under steady state of flow (Fig.2).

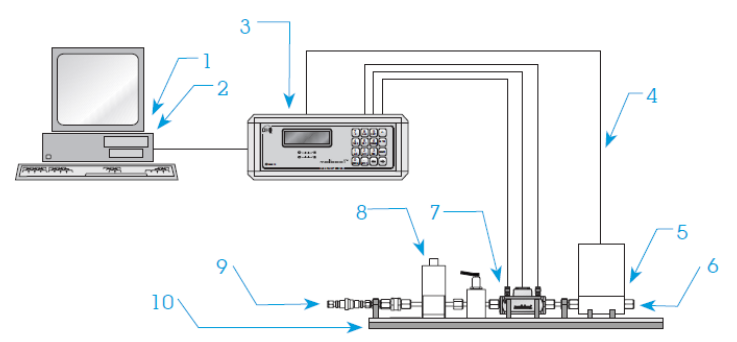

$$
\begin{aligned}
& \text { 1. Host computer with RS-232 } \\
& \text { (COM) or IEEE-488 interface } \\
& \text { 2. COMPASS for molbox, } \\
& \text { calibration software } \\
& \text { 3. molbox1 } \\
& \text { 4. molbox1 MFC control option } \\
& \text { cable }
\end{aligned}
$$

$$
\begin{aligned}
& \text { 5. Device under test, MFC } \\
& \text { 6. Atmosphere or vacuum } \\
& \text { 7. molbloc(s) } \\
& \text { 8. Regulator (molstic) } \\
& \text { 9. Gas supply } \\
& \text { 10. molstic, molbloc mounting } \\
& \text { system }
\end{aligned}
$$

Fig. 2. Calibration of thermal mass flowmeter with Molbloc/Molbox 


\subsection{Scenarios of test}

The calibration method explained in the last paragraph was used to calibrate the TMFMs that were selected and listed in table 1. The models of tested TMFMs, which are labelled from A to D to referring to the type of flowmeter and I or II to preserve the manufacturers anonymity. Six flowmeters incorporating full scales from $2 \mathrm{l}(\mathrm{n}) / \mathrm{min}$ to 100 l(n)/min. Each flowmeter was calibrated two times, the first using directly its process gas as a gas test without a correction factor, the second using a surrogate gas (air or argon) and applying the manufacturer's gas factor. Calibration tests were carried out under the same conditions of temperature and pressure. All measurements are expressed in normal conditions. The TMFMs A, B and $\mathrm{D}$ provide numerical output, while $\mathrm{C}$ is provided with analog output, 4-20 mA maximum corresponding to the full scale output, which is proportional to the measured flow. The used gases are dry air and argon with the flowing physical properties respectively (Molecular Mass:0,0289654 kg.mol ${ }^{-1}$, density: 1,293 kg.m ${ }^{-3}$ );

\begin{tabular}{|c|c|c|c|c|}
\hline Manufacturer & TMFMs & $\begin{array}{c}\text { Process } \\
\text { Gas }\end{array}$ & $\begin{array}{l}\text { Reference } \\
\text { Gas }\end{array}$ & $\begin{array}{c}\text { Full } \\
\text { scale } \\
\text { l/min }\end{array}$ \\
\hline \multirow{4}{*}{ I } & \multirow{2}{*}{ A1 } & air & air & \multirow{2}{*}{2} \\
\hline & & air & argon & \\
\hline & \multirow{2}{*}{ A2 } & argon & argon & \multirow{2}{*}{2} \\
\hline & & argon & air & \\
\hline \multirow{4}{*}{ I } & \multirow{2}{*}{ B1 } & air & air & \multirow{2}{*}{50} \\
\hline & & air & argon & \\
\hline & \multirow{2}{*}{ B2 } & argon & air & \multirow{2}{*}{50} \\
\hline & & argon & argon & \\
\hline \multirow{2}{*}{ II } & \multirow{2}{*}{$\mathrm{C}$} & air & air & \multirow{2}{*}{6} \\
\hline & & air & argon & \\
\hline \multirow{2}{*}{ II } & \multirow{2}{*}{ D } & argon & air & \multirow{2}{*}{100} \\
\hline & & argon & argon & \\
\hline
\end{tabular}

(Molecular Mass :0,039948 kg.mol ${ }^{-1}$, density: 1,784 kg. $\mathrm{m}^{-3}$ ) at $273.15 \mathrm{~K}$ and $101325 \mathrm{~Pa}$.

Table 1. Technical characteristics of the investigated TMFMs.

\section{Results and discussion}

The results of the calibration for all tests were achieved by taking multiple points, repeated three times at $10 \%$, $25 \% ; 50 \% ; 75 \%$ and $100 \%$ of the full scale flow. Fig. 3 shows the calibration results for TMFM A1. The calibration was carried out first using air as reference gas as same as the process gas. The maximum relative error for TMFM A1 is around $1,2 \%$ of the measured value, which represents $0,13 \%$ of the full scale flow. On the other hand, the calibration with a surrogate gas (argon) and using the manufacturer correction k-factor (air relative to argon calibration), the results give maximum relative error up to $4 \%$ of measured value. While the maximal tolerance error of this flow meter is $1 \%$ of full scale. The same flowmeter was tested under another configuration by changing the gas process to air and labelled TMFM A2. The results are shown in Fig.4 confirming the result obtained in the first configuration. For both scenarios we have more than $3 \%$ difference between both methods, which is considered a significant deviation in metrology. TMFM A has the same response when reference gas is air for the both configuration of process gas (argon and air). These results show that, changing the configuration doesn't have an impact on the behavior of the flowmeter.

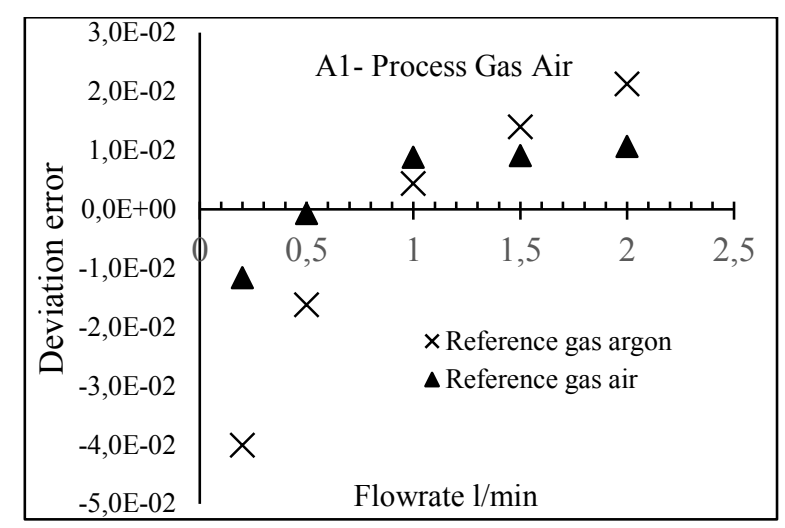

Fig. 3. The deviation error of the TMFM A1 when calibrated with Air and Argon using Gas process air.

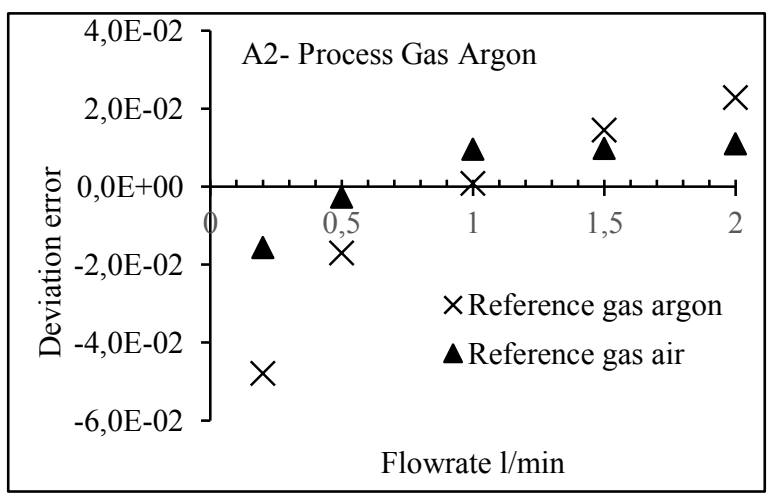

Fig. 4. The deviation error of the TMFM A2 when calibrated with Air and Argon using Gas process Argon

The same scenarios for TMFM A1, A2 were applied to calibrate TMFM B1 and B2. TMFM B has the same manufacturer but with full scale equal to $501(\mathrm{n}) / \mathrm{min}$. The results presented in both figures Fig. 5 and Fig. 6 show that the difference in deviation error between both methods represents around $1 \%$ of the measured value.

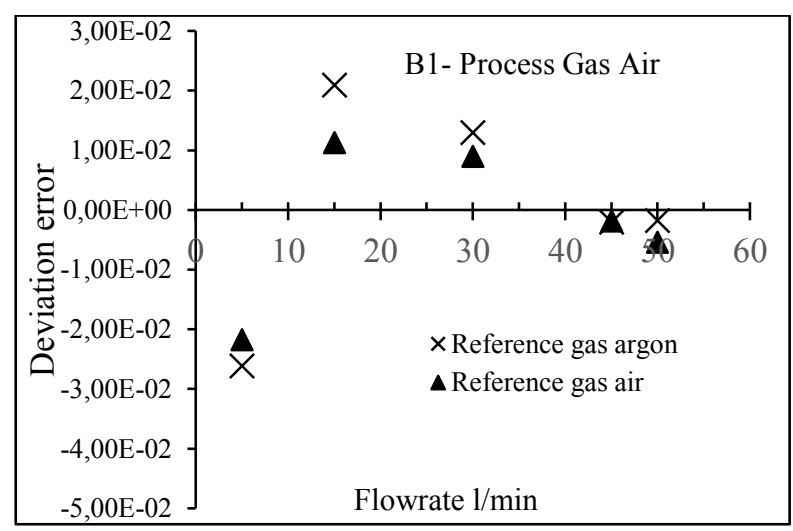

Fig. 5. The deviation error of the TMFM B1 when calibrated with Air and Argon using Gas process air 


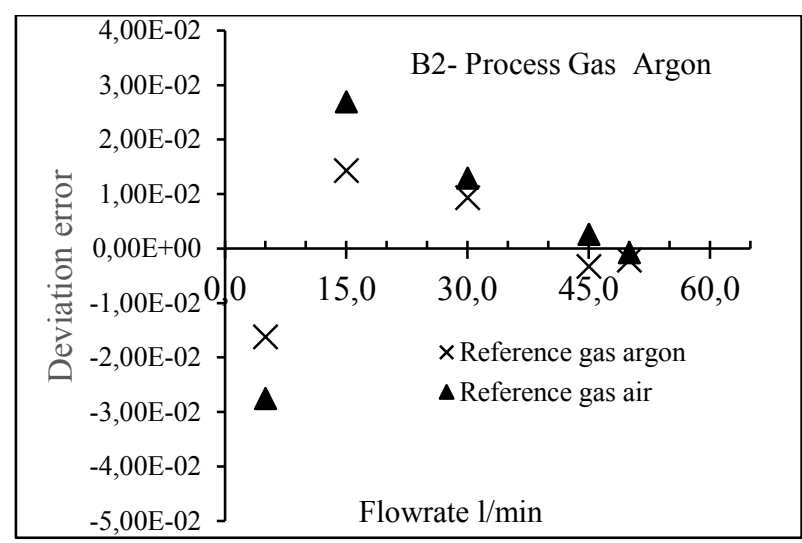

Fig. 6. The deviation error of the TMFM B2 when calibrated with Air and Argon using Gas process Argon

TMFM $\mathrm{C}$ is a flowmeter from a different manufacturer than $\mathrm{A}$ and $\mathrm{B}$. This flowmeter was tested with analog output (4-20mA). The gas process of this flowmeter is argon and there was no possibility to change the configuration as in TMFM A and B. Two scenarios of calibration were carried out. The first is using argon as reference gas and the second is using air and then the flow was corrected using the manufacturer k-factor. The results presented in Fig.7 show noticeably the different performance of the flowmeter when it is calibrated with its gas process (maximum deviation error of $1.8 \%$ of measured value and $0.6 \%$ of full scale) and when the calibration results using air as surrogate and k-factor air relative to argon (a very significant deviation error up to $16 \%)$.

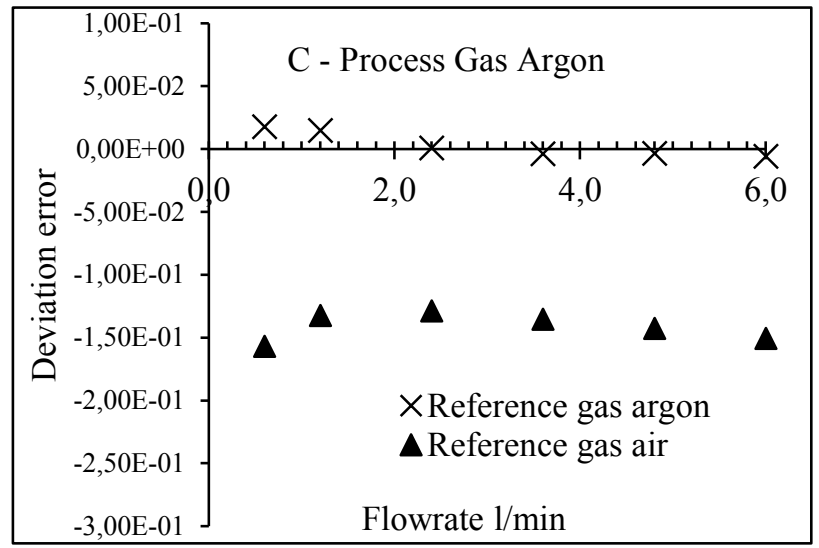

Fig. 7. The deviation error of the TMFM's $C$ when calibrated with Air and Argon using Gas process Argon

The last test was realised on TMFM D with Air gas process and $100 \mathrm{l}(\mathrm{n}) / \mathrm{min}$ of full scale. Results calibration shown in Fig. 8 confirm the results of TMFM C. The deviation error reaches $10 \%$ for the full scale using $\mathrm{k}$-factor.

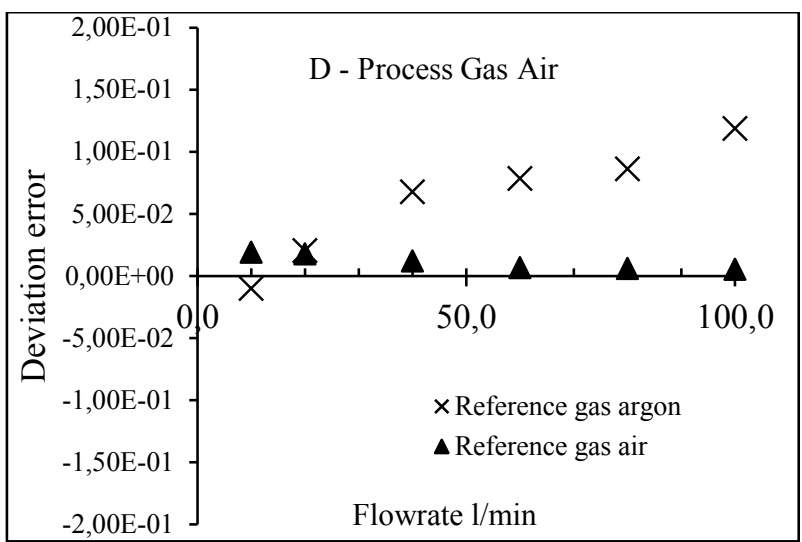

Fig. 8. The deviation error of the TMFM D when calibrated with Air and Argon using Gas process Air

\section{Conclusion}

Six TMFMs with full scale ranges of 2 1(n)/min to 100 $1(\mathrm{n}) / \mathrm{min}$ were investigated to determine their behavior when they operate with the same manufacturers gas process and also with a surrogate gas in order to confirm the reliability of the two methods.

The TMFMs were found to be well beyond their stated uncertainty as illustrated in TMFM C and D, 10\% to $16 \%$ of the measured value. When they were operated with a surrogate gas different to the manufacturers gas process. Each flowmeter was tested in the exact same laboratory condition of temperature and pressure in all scenarios, thus removing any consideration that these factors were contributory to the errors experienced. The implication of these variables could be a subject for further investigation.

This significant deviation between both calibration methods shows that the use of $\mathrm{k}$-factor introduces a systematic bias on the results. This notable bias may have an impact on the judgment on the device conformity.

These results compel the laboratory to investigate further to validate the calibration method using a surrogate gas and k-factor, as well as to study the linearity of this factor depending on flow and geometric configuration. 


\section{References}

1. S. A. Tison. A critical evaluation of thermal mass flow meters. National Institute of Standards and Technology, Gaithersburg, Maryland 20899, (1996)

2. A. Bekraoui, A. Hadjadj. An overview of thermal mass flowmeters Applicability in oil and gaz industry- Science Direct-Elsevier-Energy procedia 141-(2017),299-30.

3. ISO 14511, Measurement of fluid flow in closed conduits - Thermal mass flowmeters, second edition- ISO (2019).
4. M. Matos, V. Ferreira. Gas mass-flow meters: Principles and applications. Science Direct-Elsevier, 21 (2010) 143-149.

5. Ficco, G., Celenza, L., Dell'Isola, M., Frattolillo, A., Vigo, P., Experimental evaluation of thermal mass smart meters influence factors, Journal of Natural Gas Science \&Engineering, (2016).

6. DHI Instrument, Molbloc/Molbox Gas Flow Standards ,( 2007). 